\title{
Injective simplicial maps of the complex of arcs on nonorientable surfaces
}

\author{
ELMAS IRMAK
}

\begin{abstract}
We prove that each injective simplicial map from the complex of arcs of a compact, connected, nonorientable surface with nonempty boundary to itself is induced by a homeomorphism of the surface. We also prove that the automorphism group of the arc complex is isomorphic to the quotient of the mapping class group of the surface by its center.
\end{abstract}

57M99; 20F38

\section{Introduction}

Let $N$ be a compact, connected, nonorientable surface of genus $g$ with $r \geq 1$ boundary components. The genus of a nonorientable surface is the maximum number of projective planes in a connected sum decomposition. The mapping class group, $\operatorname{Mod}_{N}$, of $N$ is defined to be the group of isotopy classes of all self-homeomorphisms of $N$. An $\operatorname{arc} a$ on $N$ is called properly embedded if $\partial a \subseteq \partial N$ and $a$ is transverse to $\partial N$, and $a$ is called nontrivial (or essential) if $a$ cannot be deformed into $\partial N$ in such a way that the endpoints of $a$ stay in $\partial N$ during the deformation. The complex of $\operatorname{arcs}, \mathcal{A}(N)$, on $N$ is an abstract simplicial complex. Its vertices are the isotopy classes of nontrivial properly embedded arcs on $N$. A set of vertices forms a simplex if these vertices can be represented by pairwise disjoint arcs. The isotopy class of a properly embedded nontrivial arc $a$ will be denoted by $[a]$. The main results of this paper:

Theorem 1.1 Let $N$ be a compact, connected, nonorientable surface of genus $g \geq 1$ with $r \geq 1$ boundary components. If $\lambda: \mathcal{A}(N) \rightarrow \mathcal{A}(N)$ is an injective simplicial map then $\lambda$ is induced by a homeomorphism $h: N \rightarrow N$ (ie $\lambda([a])=[h(a)]$ for every vertex $[a]$ in $\mathcal{A}(N))$.

Theorem 1.2 Let $N$ be a compact, connected, nonorientable surface of genus $g \geq 1$ with $r \geq 1$ boundary components. Then $\operatorname{Aut}(\mathcal{A}(N)) \cong \operatorname{Mod}_{N} / Z\left(\operatorname{Mod}_{N}\right)$. 
Both of these theorems were proven for orientable surfaces by Irmak-McCarthy [12], and we follow the outline of this paper here. The main results in [12]: Let $R$ be a compact, connected, orientable surface of genus $g$ with $r \geq 1$ boundary components. If $\lambda: \mathcal{A}(R) \rightarrow \mathcal{A}(R)$ is an injective simplicial map then $\lambda$ is induced by a homeomorphism $h: R \rightarrow R$, and $\operatorname{Aut}(\mathcal{A}(R)) \cong \operatorname{Mod}_{R} / Z\left(\operatorname{Mod}_{R}\right)$.

Ivanov proved for orientable surfaces that the automorphisms of the complex of arcs that are induced by automorphisms of the complex of curves are induced by homeomorphisms of the surface in [13]. Using this, he proved that each automorphism of the complex of curves is induced by a homeomorphism of the surface if the genus at least two, and the automorphism group of the complex of curves is isomorphic to the mapping class group quotient by its center. As an application he proved that isomorphisms between any two finite index subgroups are geometric (ie they have the form $x \rightarrow g x g^{-1}$ for some isotopy class $g$ of a homeomorphism of the surface). Ivanov's results were proven by Korkmaz [14] and Luo [16] for lower genus cases. Luo also gave a completely different proof in all cases.

After Ivanov's work, the mapping class group was viewed as the automorphism group of various geometric objects on orientable surfaces. These objects include Schaller's complex (see Schaller [20]), the complex of pants decompositions (see Margalit [17]), the complex of nonseparating curves (see Irmak [9]), the complex of separating curves (see Brendle-Margalit [4] and McCarthy-Vautaw [18]), the Torelli geometry (see Farb-Ivanov [6]), the Hatcher-Thurston complex (see Irmak-Korkmaz [11]) and the complex of arcs (see Irmak-McCarthy [12]). As applications, Farb-Ivanov proved that the automorphism group of the Torelli subgroup is isomorphic to the mapping class group in [6], and McCarthy-Vautaw extended this result to $g \geq 3$ in [18].

Some similar results on simplicial maps and the applications for orientable surfaces are as follows: Irmak proved that superinjective simplicial maps of the complex of curves are induced by homeomorphisms of the surface if the genus is at least two in $[8 ; 10$; 9]. She used this to classify injective homomorphisms from finite index subgroups of the mapping class group to the whole group. They are geometric except in the closed genus two surface case. Behrstock-Margalit [2] and Bell-Margalit [3] proved these results for lower genus cases. Brendle-Margalit proved that superinjective simplicial maps of the separating curve complex are induced by homeomorphisms, to prove that an injection from a finite index subgroup of $K$ to the Torelli group, where $K$ is the subgroup of the mapping class group generated by Dehn twists about separating curves, is induced by a homeomorphism in $[4 ; 5]$. Shackleton proved that injective simplicial maps of the complex of curves are induced by homeomorphisms (he also considers maps between complexes of curves of different surfaces), and he obtained strong local co-Hopfian results for the mapping class groups in [20]. 
For nonorientable surfaces viewing the mapping class group as the automorphism group of some geometric objects has not been studied as much as in the orientable case. For nonorientable odd genus surfaces Atalan-Ozan proved that the automorphism group of the complex of curves is isomorphic to the mapping class group if $g+r \geq 6$ in [1]. The main results in this paper show that there are similarities to the orientable case, and we can expect more of the results listed above for the orientable case to be similar for nonorientable surfaces.

\section{Low complexity cases}

In this section we prove our main results for $(g, r)=(1,1),(g, r)=(1,2)$ and $(g, r)=(2,1)$. First we give definitions of some homeomorphisms on nonorientable surfaces and also a model of punctured $\mathbb{R P}^{2}$ as given by Korkmaz [15].

Elementary braid: If $N$ has at least two boundary components then an elementary braid is defined as follows: Let $a$ be an arc joining two distinct boundary components $\partial_{i}$ and $\partial_{j}$ of $N$. A regular neighborhood of $a \cup \partial_{i} \cup \partial_{j}$ is a pair of pants $P$. Let $z$ denote the boundary component of $P$ which is different from $\partial_{i}$ and $\partial_{j}$. Let $\sigma_{a}$ be the isotopy class of a homeomorphism $N \rightarrow N$ supported in $P$ interchanging $\partial_{i}$ and $\partial_{j}$ such that $\sigma_{a}^{2}=t_{z}$ where $t_{z}$ is the Dehn twist about $z$ (the isotopy class of a twist homeomorphism about two-sided simple closed curve $z$ ). The class $\sigma_{a}$ will be called an elementary braid.

Boundary slide: Consider a Mobius band $M$ with one hole. By sliding the boundary component along the core of $M$ we get a homeomorphism of $M$ fixing a neighborhood of $\partial M$. If $M$ is embedded in a surface $N$, this homeomorphism can be extended to a homeomorphism of $N$ by the identity. This homeomorphism and its isotopy class will be called a boundary slide.

A model of punctured $\mathbb{R P}^{2}$ : Consider the two sphere as the one point compactification of the $x y$-plane. Let $D=\left\{(x, y):(x+2)^{2}+y^{2}<1\right\}$. For a positive integer $n$, take $n$ distinct points $z_{i}$ on the line segment $x=1,-2 \leq y \leq 2$, so that $z_{1}=(1,-2), z_{n}=(1,2)$ and the second coordinates of $z_{i}$ are in ascending order. Remove the disc $D$ and identify the antipodal points on the boundary. This is a model for the surface $N$ of genus 1 with $n$ punctures as shown in Figure 1. Let $b_{j}$ be as shown in the figure. Let $v_{j}$ be the boundary slide (puncture slide) obtained by sliding $z_{j}$ once along $b_{j}$. Then $v_{j}^{2}$ is a Dehn twist about the boundary component of a regular neighborhood of $b_{j}$.

Theorem 2.1 Let $N$ be a compact, connected, nonorientable surface of genus $g$ with $r \geq 1$ boundary components. If $(g, r)=(1,1)$ or $(g, r)=(1,2)$ or $(g, r)=(2,1)$, then $\operatorname{Aut}(\mathcal{A}(N)) \cong \operatorname{Mod}_{N} / Z\left(\operatorname{Mod}_{N}\right)$. 


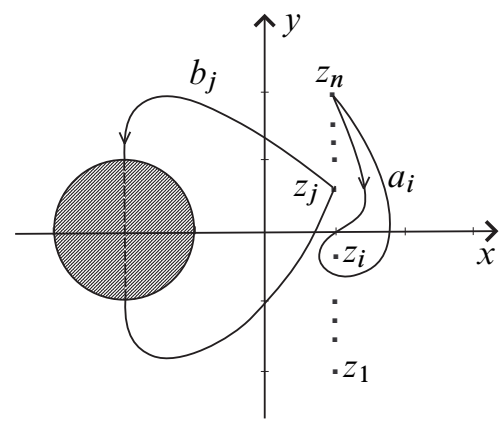

Figure 1: Punctured $\mathbb{R} \mathbb{P}^{2}$

Proof Case (i): Suppose that $(g, r)=(1,1) \cdot \operatorname{Mod}_{N}$ is isomorphic to the mapping class group of a nonorientable surface $S$ of genus 1 with 1 puncture, which is isomorphic to $\mathbb{Z}_{2}$ and it is generated by the boundary slide $v_{1}$ as defined above by Theorem 4.1 in Korkmaz [15]. So, $\operatorname{Mod}_{N} / Z\left(\operatorname{Mod}_{N}\right)$ is trivial and also $\operatorname{Aut}(\mathcal{A}(N))$ is trivial since $\mathcal{A}(N)$ has only one vertex in this case.

Case (ii): Suppose that $(g, r)=(1,2) . \operatorname{Mod}_{N}$ is isomorphic to the mapping class group of a nonorientable surface $S$ of genus 1 with 2 punctures, which is isomorphic to the Dihedral group of order 8 by Corollary 4.6 in Korkmaz [15]. It has center $\mathbb{Z}_{2}$ generated by $v_{1} v_{2}$, where $v_{i}$ is the puncture slide as defined before. We have $\operatorname{Mod}_{N} / Z\left(\operatorname{Mod}_{N}\right) \cong \mathbb{Z}_{2} \times \mathbb{Z}_{2}$. In this case $\mathcal{A}(N)$ is given by Scharlemann [20]. He proves that the complex has 8 vertices, and it is as shown in Figure 2 . It is easy to see that $\operatorname{Aut}(\mathcal{A}(N))$ is $\mathbb{Z}_{2} \times \mathbb{Z}_{2}$. Hence, we get $\operatorname{Aut}(\mathcal{A}(N)) \cong \operatorname{Mod}_{N} / Z\left(\operatorname{Mod}_{N}\right) \cong \mathbb{Z}_{2} \times \mathbb{Z}_{2}$.

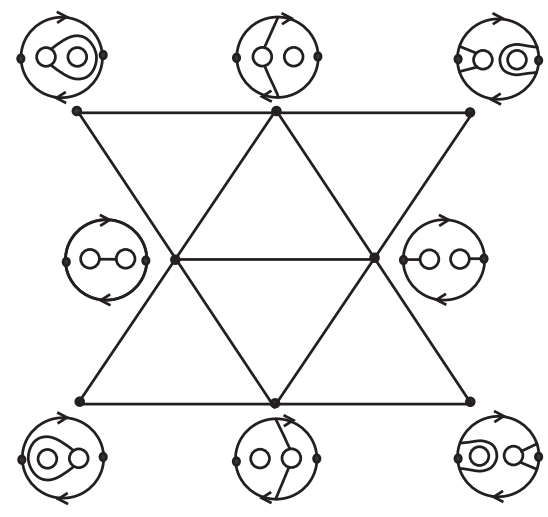

Figure 2: Complex of arcs for $(g, r)=(1,2)$ 
Case (iii): Suppose that $(g, r)=(2,1) . \operatorname{Mod}_{N}$ is isomorphic to the mapping class group of a punctured Klein bottle, and the structure of the mapping class group of $N$ is given by Stukow [21] as follows: Let $e$ and $b$ be as in the Figure 3 (i). Let $v$ be the puncture slide along $b$. Cut $N$ along $e$ to get a cylinder with one puncture. Reflection of this cylinder across the circle parallel to boundary components and passing through the puncture induces a homeomorphism $\sigma$ such that $\sigma(e)=e^{-1}$. Stukow proves that $\operatorname{Mod}_{N}=\left(\left\langle t_{e}\right\rangle \rtimes\langle v\rangle\right) \times\langle\sigma\rangle \cong\left(\mathbb{Z} \rtimes \mathbb{Z}_{2}\right) \times \mathbb{Z}_{2}$ and $Z\left(\operatorname{Mod}_{N}\right) \cong \mathbb{Z}_{2}=\langle\sigma\rangle[21$, Theorem A.5 and Corollary A.6]. So, we get $\operatorname{Mod}_{N} / Z\left(\operatorname{Mod}_{N}\right) \cong \mathbb{Z} \rtimes \mathbb{Z}_{2}$.

In this case also $\mathcal{A}(N)$ is given by Scharlemann [20]. Let $e, a, b, c$ be as in Figure 3 (i). He proves that the vertices of $\mathcal{A}(N)$ are $\left\{[a], t_{e}^{n}([b]), t_{e}^{n}([c]): n \in \mathbb{Z}\right\}$, and the complex of arcs is as shown in Figure 3 (ii). It is easy to see that the automorphism group of this complex is $\mathbb{Z} \rtimes \mathbb{Z}_{2}$. So, we get $\operatorname{Aut}(\mathcal{A}(N)) \cong \operatorname{Mod}_{N} / Z\left(\operatorname{Mod}_{N}\right) \cong \mathbb{Z} \rtimes \mathbb{Z}_{2}$.
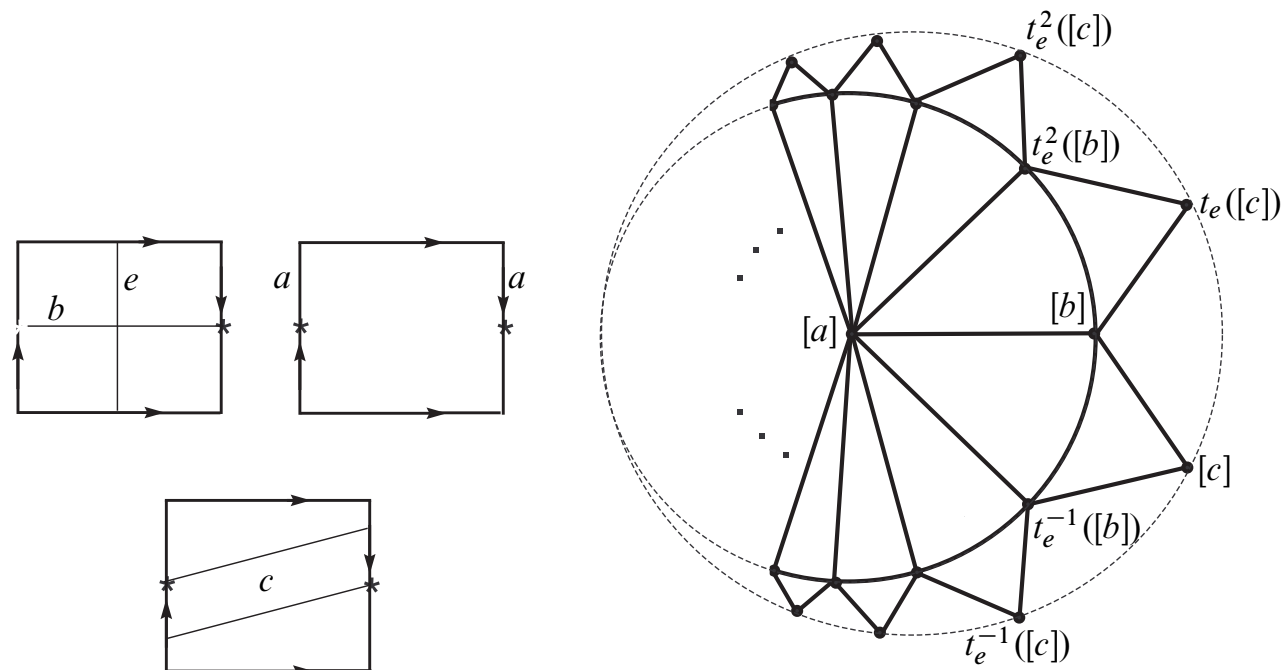

(i)

Figure 3: Complex of arcs for $(g, r)=(2,1)$

Theorem 2.2 Let $N$ be a compact, connected, nonorientable surface of genus $g$ with $r \geq 1$ boundary components. Suppose that $(g, r)=(1,1)$ or $(g, r)=(1,2)$ or $(g, r)=(2,1)$. If $\lambda: \mathcal{A}(N) \rightarrow \mathcal{A}(N)$ is an injective simplicial map then $\lambda$ is induced by a homeomorphism $h: N \rightarrow N$.

Proof For the cases $(g, r)=(1,1)$ and $(g, r)=(1,2)$, the proof follows from Theorem 2.1 as for both cases the complex of arcs has finitely many vertices so every injective simplicial map is an automorphism. 
For $(g, r)=(2,1)$, we will prove that every injection is onto. Since $[a]$ is the only vertex which is connected to infinitely many vertices in the $\mathcal{A}(N)$ and $\lambda$ is injective $[a]$ is fixed by $\lambda$. Since the elements in $A=\left\{t_{e}^{n}([b]): n \in \mathbb{Z}\right\}$ are the only vertices in $\mathcal{A}(N)$ which are connected to exactly five vertices by an edge and $\lambda$ is injective, we have $\lambda(A) \subseteq A$. Similarly, since the elements in $B=\left\{t_{e}^{n}([c]): n \in \mathbb{Z}\right\}$ are the only vertices in $\mathcal{A}(N)$ which are connected to exactly two vertices by an edge and $\lambda$ is injective, we have $\lambda(B) \subseteq B$. Let $w$ be a vertex of $\mathcal{A}(N)$ different from $[a]$. Let $\Delta$ be a top dimensional simplex containing $[a]$ in $\mathcal{A}(N)$. Since $\lambda$ is injective and $\lambda([a])=[a]$, we see that $\lambda(\Delta)$ corresponds to a top dimensional simplex, $\Delta^{\prime}$, containing $[a]$ in $\mathcal{A}(N)$. If $w$ is a vertex of $\Delta^{\prime}$, then $w$ is in the image, so we are done. Suppose that $w$ is not a vertex of $\Delta^{\prime}$. We will consider two cases:

Case (i): Suppose $w \in A$. Take a top dimensional simplex $\Delta^{\prime \prime}$ containing $w$ and $[a]$. It is easy to see that there exists a chain $\Delta^{\prime}=\Delta_{0}^{\prime}, \Delta_{1}^{\prime}, \ldots, \Delta_{n}^{\prime}=\Delta^{\prime \prime}$ of top dimensional simplices containing $a$ in $\mathcal{A}(N)$, connecting $\Delta^{\prime}$ to $\Delta^{\prime \prime}$ in such a way that the consecutive simplices $\Delta_{i}^{\prime}, \Delta_{i+1}^{\prime}$ have exactly one common face of codimension 1 . Let $\Delta_{1}, \Delta_{2}$ be two distinct top dimensional simplices in $\mathcal{A}(N)$ different from $\Delta$, such that they both contain $[a]$ and also they have one common face of codimension 1 with $\Delta$. Since $\lambda$ is injective, top dimensional simplices are sent to top dimensional simplices by $\lambda$. Every edge containing $[a]$ is contained in exactly two top dimensional simplices $\mathcal{A}(N)$. Since $\lambda([a])=[a]$ and $\lambda(\Delta)=\Delta^{\prime}$ either $\lambda\left(\Delta_{1}\right)=\Delta_{1}^{\prime}$ or $\lambda\left(\Delta_{2}\right)=\Delta_{1}^{\prime}$. This shows that every vertex of $\Delta_{1}^{\prime}$ is in the image. By an inductive argument, using the above sequence we see that $w$ is in the image of $\lambda$.

Case (ii): Suppose $w \in B$. Take a top dimensional simplex $\Delta^{\prime \prime}$ containing $w$. Let $z, t \in A$ be the distinct vertices of $\Delta^{\prime \prime}$ different from $w$. By case (i) there exist vertices $x, y \in A$ such that $z=\lambda(x), t=\lambda(y)$. Consider the top dimensional simplex $\Delta^{\prime \prime \prime}$ containing $x$ and $y$ but not $[a]$. Let $k$ the vertex of $\Delta^{\prime \prime \prime}$ different from $x$ and $y$. Since $\lambda$ is injective and $\lambda([a])=[a]$, we see that $\lambda\left(\Delta^{\prime \prime \prime}\right)$ is a top dimensional simplex containing $z$ and $t$ but not containing $[a]$. Since $\Delta^{\prime \prime}$ is the only top dimensional simplex containing $z$ and $t$ but not containing $[a]$, we see that $\Delta^{\prime \prime}=\lambda\left(\Delta^{\prime \prime \prime}\right)$ and $w=\lambda(k)$. Hence, $\lambda$ is onto. By using Theorem 2.1 we see that $\lambda$ is induced by a homeomorphism.

\section{Triangulations}

In this section we assume that $(g, r) \neq(1,1),(g, r) \neq(1,2)$ and $(g, r) \neq(2,1)$. This is equivalent to having $g+r \geq 4$ since $r \geq 1, g \geq 1$.

Let $\lambda: \mathcal{A}(N) \rightarrow \mathcal{A}(N)$ be an injective simplicial map. We will prove some properties of $\lambda$. First we give some definitions. 
Let $T$ be a set of pairwise disjoint nonisotopic arcs on $N . T$ is called a triangulation of $N$ if each component $\Delta$ of the surface $N_{T}$, obtained from $N$ by cutting $N$ along $T$, is a disc with boundary $\partial \Delta$ equal to a union of arcs, $a, b, c, d, e$, and $f$, where $a$, $b$, and $c$ correspond to elements of $T$ and $d, e$, and $f$ correspond to arcs or circles in $\partial N . \Delta$ is called a triangle of $T$, and $a, b, c$ are called sides of $\Delta$. If $a, b$, and $c$ correspond to distinct elements of $T$, then $\Delta$ is called an embedded triangle of $T$. Otherwise, $\Delta$ is called a nonembedded triangle of $T$. The phrase triangle of $T$ will also be used to refer to the image of any component $\Delta$ of $N_{T}$, under the natural quotient map $q: N_{T} \rightarrow N$, together with the images of the $\operatorname{arcs} a, b$, and $c$ of $\partial \Delta$. Two distinct triangles of a triangulation $T$ are called adjacent with respect to $T$ if they have a common side. The images of $a, b$ and $c$ will also be called sides of the triangle.

Let $T$ be a triangulation of $N$. Let $[T]$ be the set of isotopy classes of elements of $T$. Note that $[T]$ is a maximal simplex of $\mathcal{A}(N)$. Every maximal simplex $\sigma$ of $\mathcal{A}(N)$ is equal to $[T]$ for some triangulation $T$ of $N$.

Let $\lambda: \mathcal{A}(N) \rightarrow \mathcal{A}(N)$ be an injective simplicial map. Then $\lambda([T])=\left[T^{\prime}\right]$ for some triangulation $T^{\prime}$ of $N$ and $\lambda$ restricts to a correspondence $\lambda \mid:[T] \rightarrow\left[T^{\prime}\right]$ on the isotopy classes. Note that the triangulation $T^{\prime}$ of $N$ is well defined up to isotopy on $N$. By using Euler characteristic arguments we see that the number of arcs in a triangulation is $3 g+3 r-6$, and the number of triangles in a triangulation is $2 g+2 r-4$. If $\alpha$ and $\beta$ are two vertices in $\mathcal{A}(N)$, let $i(\alpha, \beta)$ denote the geometric intersection number of $\alpha$ and $\beta$, which is the minimum number of points of $a \cap b$ where $a \in \alpha$ and $b \in \beta$.

We will prove that $\lambda$ preserves geometric intersection number one property. Using this, we will prove that $\lambda$ preserves the topological equivalence of ordered triangulations on $N$ (ie for a given ordered triangulation $T=\left(a_{1}, a_{2}, \ldots, a_{3 g+3 r-6}\right)$ on $N$, and a corresponding ordered triangulation $T^{\prime}=\left(a_{1}^{\prime}, a_{2}^{\prime}, \ldots, a_{3 g+3 r-6}^{\prime}\right)$ where $\left[a_{i}^{\prime}\right]=\lambda\left(\left[a_{i}\right]\right)$ for all $i=1,2, \ldots, 3 g+3 r-6$, there exists a homeomorphism $h: N \rightarrow N$ such that $h\left(a_{i}\right)=a_{i}^{\prime}$ for all $\left.i=1,2, \ldots, 3 g+3 r-6\right)$. We will then give an argument that since $\lambda$ agrees with a homeomorphism on a given top dimensional simplex on the complex of arcs, it agrees with this homeomorphism on the whole complex.

Lemma 3.1 If $\alpha$ and $\beta$ are two vertices in $\mathcal{A}(N)$ such that $i(\alpha, \beta)=1$ then $i(\lambda(\alpha), \lambda(\beta))=1$.

Proof Let $a$ and $b$ be representatives of $\alpha$ and $\beta$ respectively such that $a$ and $b$ intersect once as in Figure 4. We complete $a$ to a triangulation $T_{1}$ of $N$ such that $\left(T_{1} \backslash\{a\}\right) \cup\{b\}$ is also a triangulation of $N$. Let $T_{2}=\left(T_{1} \backslash\{a\}\right) \cup\{b\}$. Let $\sigma_{i}$ be a simplex corresponding to triangulation $T_{i}$ for $i=1,2$. Let $\sigma_{i}^{\prime}=\lambda\left(\sigma_{i}\right)$. Since $\lambda$ is injective, $\sigma_{i}^{\prime}$ corresponds to a triangulation on $N$ for $i=1,2$. Since 


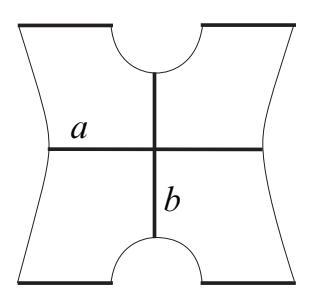

Figure 4: Arcs intersecting once

$\sigma_{1} \backslash\{\alpha\}=\sigma_{2} \backslash\{\beta\}$ and $\lambda$ is injective, we have $\sigma_{1}^{\prime} \backslash\{\lambda(\alpha)\}=\sigma_{2}^{\prime} \backslash\{\lambda(\beta)\}$. Let $T_{1}^{\prime}$ be a triangulation corresponding to $\sigma_{1}^{\prime}$. Let $a^{\prime}$ be an element of $T_{1}^{\prime}$ representing $\lambda(\alpha)$. When we delete $a^{\prime}$ from $T_{1}^{\prime}$ we get a collection of triangles and a quadrilateral. There are only two properly embedded essential nonisotopic arcs in a quadrilateral, and they intersect once. Since $\sigma_{1}^{\prime} \backslash\{\lambda(\alpha)\}=\sigma_{2}^{\prime} \backslash\{\lambda(\beta)\}$, there exists $b^{\prime} \in \lambda(\beta)$ such that $T_{1}^{\prime} \cup b^{\prime}$ is a triangulation corresponding to $\sigma_{2}^{\prime}$, and $a^{\prime}$ and $b^{\prime}$ intersect once.

Lemma 3.2 Let $a, b, c$ be properly embedded, pairwise disjoint, essential arcs on $N$ which form an embedded triangle on $N$. There exists a triangulation $T$ of $N$ containing $\{a, b, c\}$ such that the embedded triangle $\Delta$ formed by $a, b$ and $c$ on $N$ is adjacent to three distinct triangles $\Delta_{a}, \Delta_{b}$ and $\Delta_{c}$ of $T$ on $N$.

Proof Since $\Delta$ is an embedded triangle on $N$ with sides $a, b$ and $c$, these are nonisotopic essential properly embedded $\operatorname{arcs}$ on $N$. There exists a triangulation $T$ of $N$ such that $\{a, b, c\}$ is contained in $T$. Since $\Delta$ is an embedded triangle on $N$, there exist unique triangles $\Delta_{a}, \Delta_{b}$ and $\Delta_{c}$ of $T$ on $N$ which are different from $\Delta$ such that $a$ is a side of $\Delta_{a}, b$ is a side of $\Delta_{b}$ and $c$ is a side of $\Delta_{c}$. Since $(g, r) \neq(1,2)$ and $(g, r) \neq(2,1), \Delta_{a}, \Delta_{b}$ and $\Delta_{c}$ are not the same triangle on $N$.

Suppose that $\Delta_{a}=\Delta_{b}$. Then $\Delta_{a}$ has $a$ and $b$ as its sides. Since both of $a$ and $b$ are two sides of distinct triangles $\Delta_{a}$ and $\Delta, \Delta_{a}$ is an embedded triangle on $N$. Let $d$ be the third side of $\Delta_{a}$ different from $a$ and $b$. If $d=c$, then $\Delta_{a}, \Delta_{b}$ and $\Delta_{c}$ would be the same triangle on $N$, which is a contradiction. Hence, $d$ is not equal to $c$. Since $\Delta_{a}$ is an embedded triangle of $T$ on $N$ with sides $a, b$ and $d$, there exists a unique triangle $\Delta_{d}$ of $T$ on $N$ which is different from $\Delta_{a}$ and has $d$ as a side. Let $e$ and $f$ be the other sides of $\Delta_{d}$.

Suppose that $c$ is not equal to $e$ or $f$. We will consider different possible gluing of the triangles $\Delta$ and $\Delta_{a}$. Suppose the gluing is as shown in the first part of Figure 5. The sides of $\Delta_{d}$ are $d, e$ and $f$, none of which are equal to $c$. Since $c$ is a side of $\Delta_{c}$, $\Delta_{c}$ and $\Delta_{d}$ are distinct triangles of $T$. Since $\Delta_{a}$ and $\Delta_{d}$ are distinct triangles of $T$ 

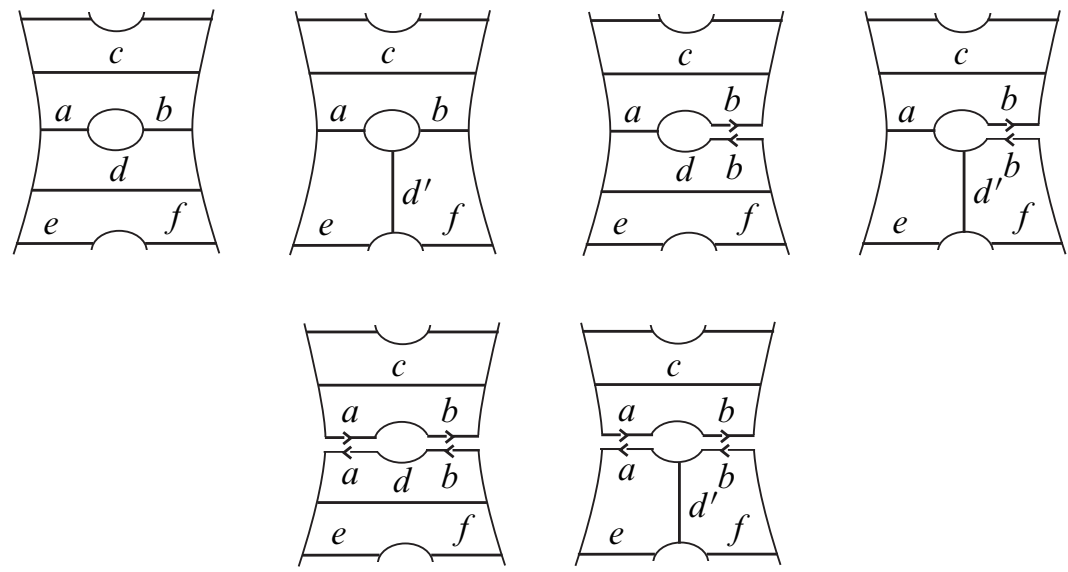

Figure 5: Obtaining four triangles by one elementary move

having $d$ as a side, there is a quadrilateral $\Omega$ on $N$ with sides $a, b, e$ and $f$, and diagonal $d$ as shown in the figure. Let $d^{\prime}$ be as shown in the second part of Figure 5. Let $T^{\prime}=(T \backslash\{d\}) \cup\left\{d^{\prime}\right\}$ be the triangulation on $N$ which is obtained from the triangulation $T$ on $N$ by an elementary move replacing $d$ with $d^{\prime}$. The triangulation $T^{\prime}$ has the properties that we wanted. It contains $\{a, b, c\}$, and the embedded triangle $\Delta$ formed by $a, b$ and $c$ on $N$ is adjacent to three distinct triangles of $T^{\prime}$ on $N$. In Figure 5 we show how to choose $d^{\prime}$ for the other possible different gluing of the two triangles $\Delta$ and $\Delta_{a}$. Similarly, in all cases we get a desired triangulation.

Suppose that $c$ is equal to either $e$ or $f$. We may assume that $c=e$. We have that $\Delta_{d}$ is an embedded triangle of $T$ on $N$ with sides $c, d$ and $f$, where $f$ is some element of $T$ which is not equal to $a, b, c$ or $d$. We will consider different possible gluing of the triangles $\Delta, \Delta_{a}$ and $\Delta_{d}$. Suppose the gluing is as shown in the first part of Figure 6. Since $f$ is a side of the embedded triangle $\Delta_{d}$ of $T$ on $N$, there is a
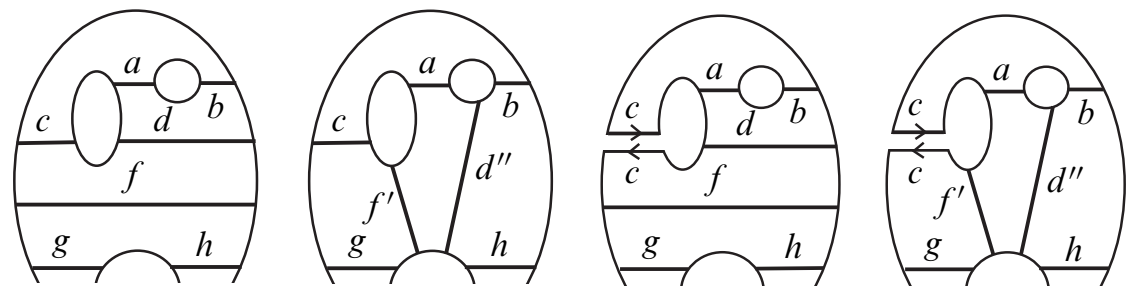

Figure 6: Obtaining four triangles by two elementary moves 
unique triangle $\Delta_{f}$ of $T$ on $N$ which is distinct from $\Delta_{d}$ and has $f$ as a side. Let $g$ and $h$ be the other sides of $\Delta_{f}$. Let $T^{\prime}$ be the triangulation obtained from $T$ by an elementary move replacing the element $f$ of $T$ by $f^{\prime}$ as shown in the figure. Let $T^{\prime \prime}$ be the triangulation obtained from $T^{\prime}$ by an elementary move replacing the element $d$ of $T^{\prime}$ by $d^{\prime \prime}$ as shown in the second part of Figure 6. The triangulation $T^{\prime \prime}$ has the properties that we wanted. It contains $\{a, b, c\}$, and the embedded triangle $\Delta$ formed by $a, b$ and $c$ on $N$ is adjacent to three distinct triangles of $T^{\prime \prime}$ on $N$. In Figure 6 we show how to choose $f^{\prime}$ and $d^{\prime \prime}$ for another possible gluing of the triangles $\Delta, \Delta_{a}$ and $\Delta_{d}$. We note that similarly in the other possible cases of gluing we can choose $f^{\prime}$ and $d^{\prime \prime}$ and get a triangulation $T^{\prime \prime}$ that has the properties that we want.

This shows that in any case there is a triangulation of $N$ with the desired properties. $\square$

Lemma 3.3 Let $a, b, c$ be properly embedded, pairwise disjoint, essential arcs on $N$ which form an embedded triangle on $N$. Then there exist $a^{\prime} \in \lambda([a]), b^{\prime} \in \lambda([b]), c^{\prime} \in$ $\lambda([c])$ such that $a^{\prime}, b^{\prime}, c^{\prime}$ form an embedded triangle on $N$.

Proof Since $g+r \geq 4$, by Lemma 3.2 there exists a triangulation $T$ of $N$ containing $\{a, b, c\}$ such that the embedded triangle $\Delta$ formed by $a, b$ and $c$ on $N$ is adjacent to three distinct triangles $\Delta_{a}, \Delta_{b}$ and $\Delta_{c}$ of $T$ on $N$.

Note that $\partial \Delta_{a}$ is equal to union of $\operatorname{arcs} a_{1}, x_{1}, a_{2}, x_{2}, a_{3}, x_{3}$ where $a_{1}, a_{2}, a_{3}$ correspond to elements of $T$ and each of $x_{1}, x_{2}, x_{3}$ correspond to an arc or a component of $\partial R$. Without loss of generality, we assume that $a_{1}$ corresponds to $a$ and $x_{2}$ is disjoint from $a_{1}$. Similarly, $\partial \Delta_{b}$ is equal to union of $\operatorname{arcs} b_{1}, y_{1}, b_{2}, y_{2}, b_{3}, y_{3}$ where $b_{1}, b_{2}, b_{3}$ correspond to elements of $T$ and each of $y_{1}, y_{2}, y_{3}$ correspond to an arc or a component of $\partial R$. Without loss of generality, we assume that $b_{1}$ corresponds to $b$ and $y_{2}$ is disjoint from $b_{1}$. Likewise, $\partial \Delta_{c}$ is equal to union of $\operatorname{arcs} c_{1}, z_{1}, c_{2}, z_{2}, c_{3}, z_{3}$ where $c_{1}, c_{2}, c_{3}$ correspond to elements of $T$ and each of $z_{1}, z_{2}, z_{3}$ correspond to an arc or a component of $\partial R$. Without loss of generality, we assume that $c_{1}$ corresponds to $c$ and $z_{2}$ is disjoint from $c_{1}$.

Let $p$ be a properly embedded, essential arc connecting $x_{2}$ to $y_{2}$ and intersecting only $a$ and $b$ once and disjoint from each element of $T \backslash\{a, b\}$ as shown in Figure 7 (i). Let $q$ be a properly embedded, essential arc connecting $y_{2}$ to $z_{2}$ and intersecting only $b$ and $c$ once and disjoint from $p$ and each element of $T \backslash\{b, c\}$ as shown in Figure 7 (i). Let $s$ be a properly embedded, essential arc connecting $x_{2}$ to $z_{2}$ and intersecting only $a$ and $c$ once and disjoint from $p \cup q$ and each element of $T \backslash\{a, c\}$ as shown in Figure 7 (i). The arcs $p, q$ and $s$ form an embedded triangle on $N$. It is easy to see that $T_{1}=(T \backslash\{a, b, c\}) \cup\{p, q, s\}$ is a triangulation on $N$. 


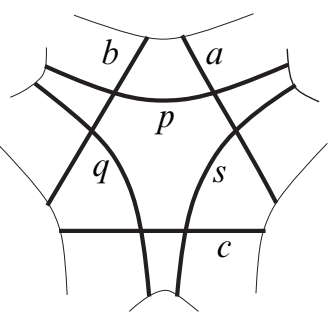

(i)

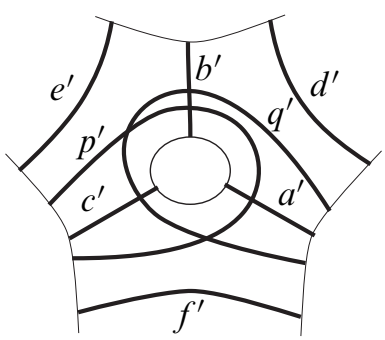

(ii)

Figure 7: Embedded triangle I

Let $T^{\prime}$ be a triangulation on $N$ such that $\lambda([T])=\left[T^{\prime}\right]$. Let $a^{\prime}, b^{\prime}, c^{\prime}$ be representatives of $\lambda([a]), \lambda([b]), \lambda([c])$ respectively, such that they are also in $T^{\prime}$.

We have $i([p],[w])=0$ for every element $w \in T \backslash\{a, b\}, i([p],[a])=1$ and $i([p],[b])=$ 1. Since $\lambda$ is injective, by using Lemma 3.1 we see that $i(\lambda([p]), \lambda([w]))=0$ for every element $w \in T \backslash\{a, b\}, i(\lambda([p]), \lambda([a]))=1$ and $i(\lambda([p]), \lambda([b]))=1$. Hence, there exists $p^{\prime} \in \lambda([p])$ such that $p^{\prime}$ intersects $a^{\prime}$ and $b^{\prime}$ essentially once and does not intersect any other element of $T^{\prime}$. Similarly, there exists $q^{\prime} \in \lambda([q])$ such that $q^{\prime}$ intersects $b^{\prime}$ and $c^{\prime}$ essentially once and does not intersect any other element of $T^{\prime}$, and there exists $s^{\prime} \in \lambda([s])$ such that $s^{\prime}$ intersects $a^{\prime}$ and $c^{\prime}$ essentially once and does not intersect any other element of $T^{\prime}$.

Since $p, q$, and $s$ are disjoint, $i([p],[q])=i([q],[s])=i([s],[p])=0$. Since $\lambda$ is a simplicial map, it follows that $i(\lambda([p]), \lambda([q]))=i(\lambda([q]), \lambda([s]))=i(\lambda([s]), \lambda([p]))=$ 0 . Hence, we may assume that $p^{\prime}, q^{\prime}$, and $s^{\prime}$ are pairwise disjoint arcs on $N$.

Since the essential arc $p^{\prime}$ on $N$ intersects $a^{\prime}$ and $b^{\prime}$ essentially once and is disjoint from the other elements of the triangulation $T^{\prime}$ of $N$, there exists a triangle $\Delta_{1}$ of $T^{\prime}$ on $N$ having sides corresponding to $a^{\prime}$ and $b^{\prime}$. Similarly, there exists a triangle $\Delta_{2}$ of $T^{\prime}$ on $N$ having sides corresponding to $b^{\prime}$ and $c^{\prime}$, and a triangle $\Delta_{3}$ of $T^{\prime}$ on $N$ having sides corresponding to $c^{\prime}$ and $a^{\prime}$. Let $d^{\prime}$ be the side of $\Delta_{1}$ different from $a^{\prime}, b^{\prime}$ in $T^{\prime}$, let $e^{\prime}$ be the side of $\Delta_{2}$ different from $b^{\prime}, c^{\prime}$ in $T^{\prime}$. Let $f^{\prime}$ be the side of $\Delta_{3}$ different from $a^{\prime}, c^{\prime}$ in $T^{\prime}$.

If $d^{\prime}=c^{\prime}$ or $e^{\prime}=a^{\prime}$ or $f^{\prime}=b^{\prime}$, then $a^{\prime}, b^{\prime}, c^{\prime}$ form an embedded triangle, so we are done. Assume that $d^{\prime} \neq c^{\prime}, e^{\prime} \neq a^{\prime}$, and $f^{\prime} \neq b^{\prime}$. Since $a^{\prime}, b^{\prime}$, and $c^{\prime}$ are distinct arcs on $N$, we have $\Delta_{1} \neq \Delta_{2}, \Delta_{2} \neq \Delta_{3}$ and $\Delta_{3} \neq \Delta_{1}$. Hence, $\Delta_{1}, \Delta_{2}$, and $\Delta_{3}$ are three distinct components of $N_{T^{\prime}}$. 
We need to consider cases depending on the placement of the $\operatorname{arcs} c^{\prime}, e^{\prime}$, and $f^{\prime}$ on $\partial \Delta_{2}$ and $\partial \Delta_{3}$ and the possible gluing of the triangles $\Delta_{1}, \Delta_{2}, \Delta_{3}$ on $N$. These cases are shown in Figures 7 (ii), 8, 9 and 10.

Case (i): Assume $a^{\prime}, b^{\prime}, c^{\prime}, d^{\prime}, e^{\prime}, f^{\prime}$ are as shown in Figure 7 (ii). Note that the arc $p^{\prime}$ on $N$ representing $\lambda([p])$ intersects $b^{\prime}$ and $a^{\prime}$ once essentially and is disjoint from $e^{\prime}, d^{\prime}, f^{\prime}$, and $c^{\prime}$; and the arc $q^{\prime}$ on $N$ representing $\lambda([q])$ intersects $b^{\prime}$ and $c^{\prime}$ once essentially and is disjoint from $e^{\prime}, d^{\prime}, f^{\prime}$, and $a^{\prime}$. But then we see that $p^{\prime}$ and $q^{\prime}$ intersect essentially, which gives a contradiction since $i(\lambda([p]), \lambda([q]))=0$. Similarly, we can show that if $a^{\prime}, b^{\prime}, c^{\prime}, d^{\prime}, e^{\prime}, f^{\prime}$ are as shown in Figure 8 (i) or (ii) or (iii) we get a contradiction (see Figure 8).

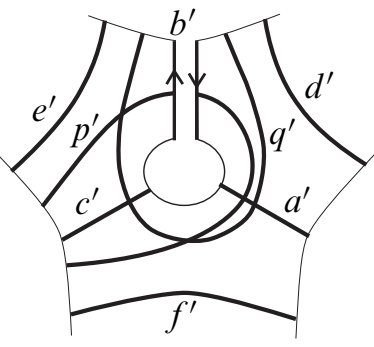

(i)

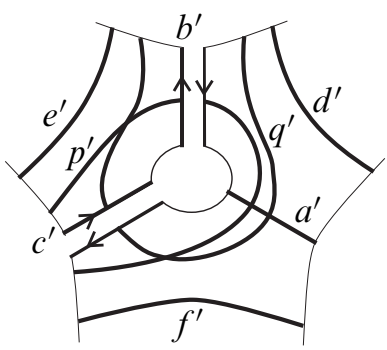

(ii)

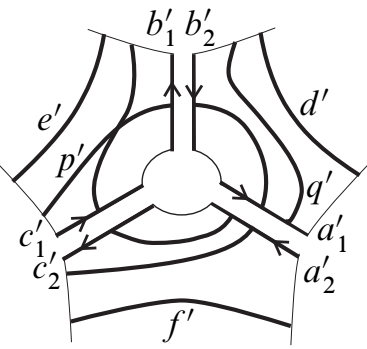

(iii)

Figure 8: Embedded triangle II

Case (ii): Assume $a^{\prime}, b^{\prime}, c^{\prime}, d^{\prime}, e^{\prime}, f^{\prime}$ are as shown in Figure 9 (i). As before, it follows that the arc $p^{\prime}$ on $N$ representing $\lambda([p])$ intersects $b^{\prime}$ and $a^{\prime}$ once essentially and is disjoint from $e^{\prime}, d^{\prime}, f^{\prime}$, and $c^{\prime}$; and the $\operatorname{arc} s^{\prime}$ on $N$ representing $\lambda([s])$ intersects $a^{\prime}$ and $c^{\prime}$ once essentially and is disjoint from $e^{\prime}, d^{\prime}, f^{\prime}, b^{\prime}$. But then we see that $p^{\prime}$ and $s^{\prime}$ intersect essentially which gives a contradiction, since $i(\lambda([p]), \lambda([s]))=0$. Similarly, we can show that if $a^{\prime}, b^{\prime}, c^{\prime}, d^{\prime}, e^{\prime}, f^{\prime}$ are as shown in the remaining parts of Figure 9 we get a contradiction.

Case (iii): If $a^{\prime}, b^{\prime}, c^{\prime}, d^{\prime}, e^{\prime}, f^{\prime}$ are as shown in Figure 10, we get a contradiction as in the proof Case (ii). In the remaining cases, where the placement of the arcs on the triangles are as in Figure 10, we can see all possible gluing of these triangles formed by $e^{\prime}, b^{\prime}, c^{\prime}$ and $a^{\prime}, c^{\prime}, f^{\prime}$ and $a^{\prime}, b^{\prime}, d^{\prime}$ as we saw in Figure 9, and get contradictions similarly as in Case (ii).

Hence, we see that either $d^{\prime}=c^{\prime}$ or $e^{\prime}=a^{\prime}$ or $f^{\prime}=b^{\prime}$ and, hence, we are done. 

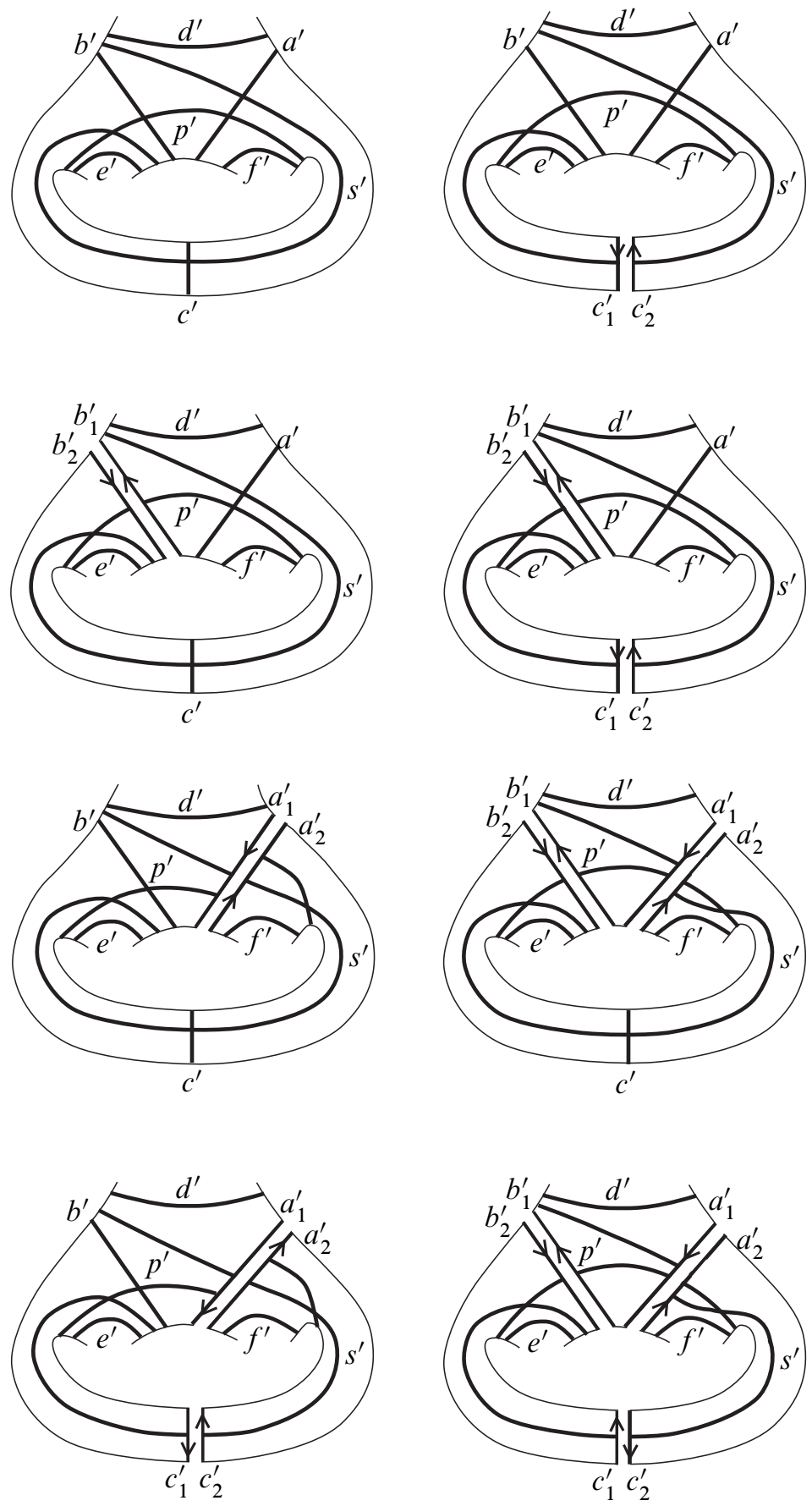

Figure 9: Embedded triangle III 


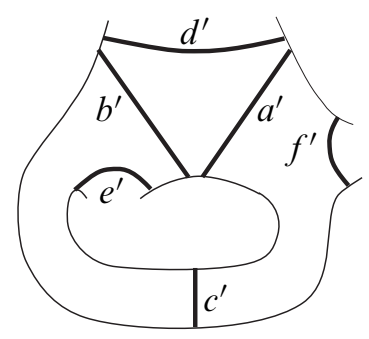

Figure 10: Embedded triangle IV

A nonembedded triangle is called a regular nonembedded triangle if it is homeomorphic to an annulus on the surface. In Figure 11 (i) we see a regular nonembedded triangle formed by $a$ and $b$. A nonembedded triangle is called a twisted nonembedded triangle if it is homeomorphic to a Mobius band on the surface. In Figure 11 (ii) we see a twisted nonembedded triangle formed by $a$ and $b$.

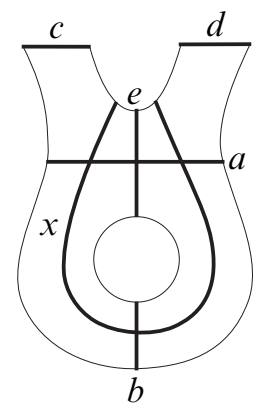

Figure 11: (i) Arc configurations I

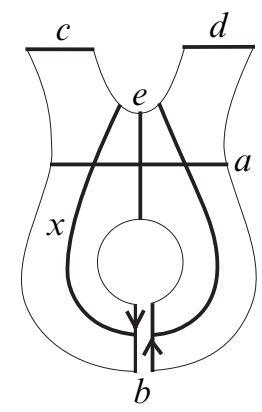

(ii) Arc configurations II

Lemma 3.4 Let $a, b$ be properly embedded, disjoint, essential arcs on $N$ such that $a$ connects one boundary component to itself, and $a, b$ form a regular nonembedded triangle on $N$. There exist $a^{\prime} \in \lambda([a])$ and $b^{\prime} \in \lambda([b])$ such that $a^{\prime}$ connects one boundary component to itself, and $a^{\prime}, b^{\prime}$ form a regular nonembedded triangle on $N$.

Proof We complete $a$ and $b$ to an arc configuration consisting of $\operatorname{arcs}\{a, b, c, d, e, x\}$ as shown in Figure 11 (i). By using Lemma 3.3 we can see that since $b, c, d, e$ are pairwise disjoint essential arcs and $b, c, e$ and $b, d, e$ form embedded triangles on $N$ there exist $b^{\prime} \in \lambda([b]), c^{\prime} \in \lambda([c]), d^{\prime} \in \lambda([d]), e^{\prime} \in \lambda([e])$ such that $b^{\prime}, c^{\prime}, d^{\prime}, e^{\prime}$ are pairwise disjoint and $b^{\prime}, c^{\prime}, e^{\prime}$ and $b^{\prime}, d^{\prime}, e^{\prime}$ form embedded triangles on $N$. Since $a$ intersects $e$ once and $a$ is disjoint from each of $b, c, d$, by using Lemma 3.1 we 
can choose $a^{\prime} \in \lambda([a])$ such that $a^{\prime}$ intersects $e^{\prime}$ once and $a^{\prime}$ is disjoint from each of $b^{\prime}, c^{\prime}, d^{\prime}$. Since $a, c, d$ form an embedded triangle $a^{\prime}, c^{\prime}, d^{\prime}$ form an embedded triangle.

Now there are four cases to consider as shown in Figure 12:

Case (i): In the first case $a^{\prime}, b^{\prime}$ form a regular nonembedded triangle on $N$.

Case (ii): Suppose the gluing of the triangles formed by $b^{\prime}, c^{\prime}, e^{\prime}$ and $b^{\prime}, d^{\prime}, e^{\prime}$ are as shown in 12 (ii). Then $a^{\prime}, c^{\prime}, d^{\prime}$ do not form an embedded triangle as shown in the figure which gives a contradiction.

Case (iii): Suppose the gluing of the triangles formed by $b^{\prime}, c^{\prime}, d^{\prime}$ and $a^{\prime}, b^{\prime}, c^{\prime}$ are as shown in 12 (iii). We consider the arc $x$ intersecting $b$ once and being disjoint from each of $c, d, e$. By using Lemma 3.1 we can choose $x^{\prime} \in \lambda([x])$ such that $x^{\prime}$ intersects $b^{\prime}$ once and $x^{\prime}$ is disjoint from each of $c^{\prime}, d^{\prime}, e^{\prime}$. By using Lemma 3.3 we get a contradiction as $c, x, d$ form an embedded triangle but $c^{\prime}, x^{\prime}, d^{\prime}$ do not form an embedded triangle on $N$ as shown in Figure 12 (iii).

Case (iv): We get a contradiction as in Case (iii).

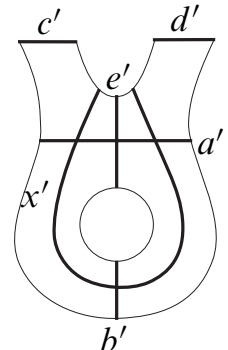

(i)

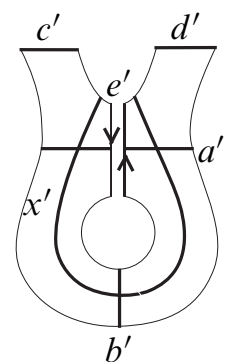

(ii)

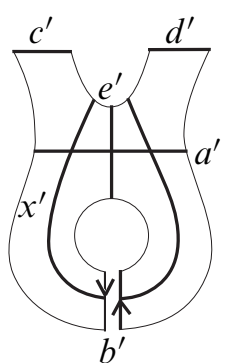

(iii)

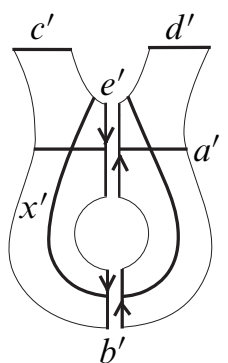

(iv)

Figure 12: Arc configurations III

Lemma 3.5 Let $a, b$ be properly embedded, disjoint, essential arcs on $N$ such that $a$ connects one boundary component to itself, and $a, b$ form a twisted nonembedded triangle on $N$. There exist $a^{\prime} \in \lambda([a])$ and $b^{\prime} \in \lambda([b])$ such that $a^{\prime}$ connects one boundary component to itself and $a^{\prime}, b^{\prime}$ form a twisted nonembedded triangle on $N$.

Proof We complete $a$ and $b$ to an arc configuration consisting of $\operatorname{arcs}\{a, b, c, d, e, x\}$ as shown in Figure 11 (ii). By using Lemma 3.3 we can see that since $b, c, d, e$ are pairwise disjoint essential arcs and $b, c, e$ and $b, d, e$ form embedded triangles on $N$ 
there exist $b^{\prime} \in \lambda([b]), c^{\prime} \in \lambda([c]), d^{\prime} \in \lambda([d]), e^{\prime} \in \lambda([e])$ such that $b^{\prime}, c^{\prime}, d^{\prime}, e^{\prime}$ are pairwise disjoint and $b^{\prime}, c^{\prime}, e^{\prime}$ and $b^{\prime}, d^{\prime}, e^{\prime}$ form embedded triangles on $N$. Since $a$ intersects $e$ once and $a$ is disjoint from each of $b, c, d$, by using Lemma 3.1 we can choose $a^{\prime} \in \lambda([a])$ such that $a^{\prime}$ intersects $e^{\prime}$ once and $a^{\prime}$ is disjoint from each of $b^{\prime}, c^{\prime}, d^{\prime}$. Since $a, c, d$ form an embedded triangle $a^{\prime}, c^{\prime}, d^{\prime}$ form an embedded triangle.

Now there are four cases to consider as shown in Figure 12:

Case (i): Suppose the gluing of the triangles formed by $b^{\prime}, c^{\prime}, e^{\prime}$ and $b^{\prime}, d^{\prime}, e^{\prime}$ are as shown in Figure 12 (i). We consider the arc $x$ intersecting $b$ once and being disjoint from each of $c, d, e$. By using Lemma 3.1 we can choose $x^{\prime} \in \lambda([x])$ such that $x^{\prime}$ intersects $b^{\prime}$ once and $x^{\prime}$ is disjoint from each of $c^{\prime}, d^{\prime}, e^{\prime}$. By using Lemma 3.3 we get a contradiction as $c, x$,e form an embedded triangle but $c^{\prime}, x^{\prime}, e^{\prime}$ do not form an embedded triangle on $N$ as shown in Figure 11 (ii) and Figure 12 (i).

Case (ii): Suppose the gluing of the triangles formed by $b^{\prime}, c^{\prime}, d^{\prime}$ and $a^{\prime}, b^{\prime}, c^{\prime}$ are as shown in 12 (ii). We get a contradiction as in Case (i).

Case (iii) In this case $a^{\prime}, b^{\prime}$ form a twisted nonembedded triangle on $N$.

Case (iv): Suppose the gluing of the triangles formed by $b^{\prime}, c^{\prime}, e^{\prime}$ and $b^{\prime}, d^{\prime}, e^{\prime}$ are as shown in 12 (iv). We see that $a^{\prime}, c^{\prime}, d^{\prime}$ do not form an embedded triangle as shown in the figure which gives a contradiction.

Lemma 3.6 Let $a, b, c, d$, e be essential, pairwise disjoint, nonisotopic, properly embedded arcs on $N$. Suppose there is a subsurface $K \subseteq N$ and a homeomorphism $\phi:(K, a, b, c, d, e) \rightarrow\left(K_{0}, a_{0}, b_{0}, c_{0}, d_{0}, e_{0}\right)$ where $K_{0}, a_{0}, b_{0}, c_{0}, d_{0}, e_{0}$ are shown in Figure 13 (i). There exist $a^{\prime} \in \lambda([a]), b^{\prime} \in \lambda([b]), c^{\prime} \in \lambda([c]), d^{\prime} \in \lambda([d]), e^{\prime} \in \lambda([e])$, $K^{\prime} \subseteq N$ and a homeomorphism $\chi:\left(K^{\prime}, a^{\prime}, b^{\prime}, c^{\prime}, d^{\prime}, e^{\prime}\right) \rightarrow\left(K_{0}, a_{0}, b_{0}, c_{0}, d_{0}, e_{0}\right)$.

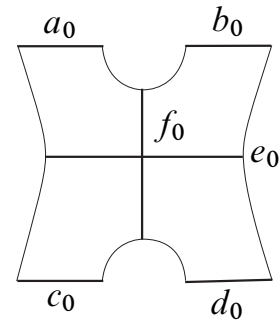

(i)

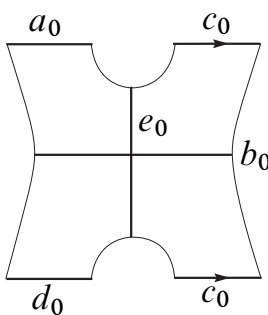

(ii)

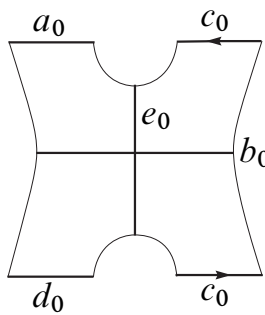

(iii)

Figure 13: Arc configurations IV 
Proof Let $f_{0}$ be as shown in Figure 13 (i). Let $f=\phi^{-1}\left(f_{0}\right)$. We see that $f$ is an essential, properly embedded arc on $N$ such that $f$ intersects $e$ once and $f$ is disjoint from $a, b, c, d$. Since $a, b, c, d, e$ are disjoint, $a, b, e$ and $c, d, e$ form embedded triangles, by using that $\lambda$ is injective and the results of Lemma 3.3, we can choose $a^{\prime} \in \lambda([a]), b^{\prime} \in \lambda([b]), c^{\prime} \in \lambda([c]), d^{\prime} \in \lambda([d]), e^{\prime} \in \lambda([e])$ such that $a^{\prime}, b^{\prime}, c^{\prime}, d^{\prime}, e^{\prime}$ are disjoint, and $a^{\prime}, b^{\prime}, e^{\prime}$ and $c^{\prime}, d^{\prime}, e^{\prime}$ form embedded triangles. Since $e, f$ intersect once and $f$ is disjoint from each of $a, b, c, d$, we can choose $f^{\prime} \in \lambda([f])$ such that $e^{\prime}, f^{\prime}$ intersect once and $f^{\prime}$ is disjoint from each of $a^{\prime}, b^{\prime}, c^{\prime}, d^{\prime}$. Since $a, c, f$ form an embedded triangle, $a^{\prime}, c^{\prime}, f^{\prime}$ form an embedded triangle. Then the result of the lemma follows.

Lemma 3.7 Let $a, b, c, d$ be essential, pairwise disjoint, nonisotopic, properly embedded $\operatorname{arcs}$ on $N$. Suppose that there exists a subsurface $K$ of $N$ and a homeomorphism $\phi:(K, a, b, c, d) \rightarrow\left(K_{0}, a_{0}, b_{0}, c_{0}, d_{0}\right)$ where $K_{0}$ and $a_{0}, b_{0}, c_{0}, d_{0}$ are as shown in Figure 13 (ii). There exist $a^{\prime} \in \lambda([a]), b^{\prime} \in \lambda([b]), c^{\prime} \in \lambda([c]), d^{\prime} \in \lambda([d]), K^{\prime} \subseteq N$ and a homeomorphism $\chi:\left(K^{\prime}, a^{\prime}, b^{\prime}, c^{\prime}, d^{\prime}\right) \rightarrow\left(K_{0}, a_{0}, b_{0}, c_{0}, d_{0}\right)$.

Proof Let $e_{0}$ be as shown in Figure 13 (ii). Let $e=\phi^{-1}\left(e_{0}\right)$. We see that $e$ is an essential, properly embedded arc on $N$ such that $e$ intersects $b$ once and $e$ is disjoint from $a, c, d$. Since $a, c, d, e$ are disjoint, $e, c$ form a regular nonembedded triangle, $a, d, e$ form an embedded triangle, by using that $\lambda$ is injective and the results of Lemma 3.3 and Lemma 3.4, we can choose $a^{\prime} \in \lambda([a]), e^{\prime} \in \lambda([e]), c^{\prime} \in \lambda([c]), d^{\prime} \in \lambda([d])$ such that $a^{\prime}, c^{\prime}, d^{\prime}, e^{\prime}$ are disjoint, $e^{\prime}, c^{\prime}$ form a regular nonembedded triangle and $a^{\prime}, d^{\prime}, e^{\prime}$ form an embedded triangle. Since $e, b$ intersect once and $e$ is disjoint from each of $a, c, d$, we can choose $b^{\prime} \in \lambda([b])$ such that $e^{\prime}, b^{\prime}$ intersect once and $e^{\prime}$ is disjoint from each of $a^{\prime}, c^{\prime}, d^{\prime}$, then the result of the lemma follows.

Lemma 3.8 Let $a, b, c, d$ be essential, pairwise disjoint, nonisotopic, properly embedded $\operatorname{arcs}$ on $N$. Suppose that there exists a subsurface $K$ of $N$ and a homeomorphism $\phi:(K, a, b, c, d) \rightarrow\left(K_{0}, a_{0}, b_{0}, c_{0}, d_{0}\right)$ where $K_{0}$ and $a_{0}, b_{0}, c_{0}, d_{0}$ are as shown in Figure 13 (iii). Then there exist $a^{\prime} \in \lambda([a]), b^{\prime} \in \lambda([b]), c^{\prime} \in \lambda([c]), d^{\prime} \in \lambda([d]), K^{\prime} \subseteq N$ and a homeomorphism $\chi:\left(K^{\prime}, a^{\prime}, b^{\prime}, c^{\prime}, d^{\prime}\right) \rightarrow\left(K_{0}, a_{0}, b_{0}, c_{0}, d_{0}\right)$.

Proof Let $e_{0}$ be as shown in Figure 13 (iii). Let $e=\phi^{-1}\left(e_{0}\right)$. We see that $e$ is an essential, properly embedded arc on $N$ such that $e$ intersects $b$ once and $e$ is disjoint from $a, c, d$. Since $a, c, d, e$ are disjoint, $e, c$ form a twisted nonembedded triangle, $a, d, e$ form an embedded triangle, by using that $\lambda$ is injective and the results of Lemma 3.3 and Lemma 3.5, we can choose $a^{\prime} \in \lambda([a]), e^{\prime} \in \lambda([e]), c^{\prime} \in \lambda([c]), d^{\prime} \in \lambda([d])$ such that $a^{\prime}, c^{\prime}, d^{\prime}, e^{\prime}$ are disjoint, $e^{\prime}, c^{\prime}$ form a twisted nonembedded triangle and $a^{\prime}, d^{\prime}, e^{\prime}$ 
form an embedded triangle. Since $e, b$ intersect once and $e$ is disjoint from each of $a, c, d$, we can choose $b^{\prime} \in \lambda([b])$ such that $e^{\prime}, b^{\prime}$ intersect once and $e^{\prime}$ is disjoint from each of $a^{\prime}, c^{\prime}, d^{\prime}$, then the result of the lemma follows.

Lemma 3.9 Let $a, b, c, d$ be essential, pairwise disjoint, nonisotopic, properly embedded $\operatorname{arcs}$ on $N$. Suppose that there exists a subsurface $K$ of $N$ and a homeomorphism $\phi:(K, a, b, c, d) \rightarrow\left(K_{0}, a_{0}, b_{0}, c_{0}, d_{0}\right)$ where $K_{0}$ and $a_{0}, b_{0}, c_{0}, d_{0}$ are as shown in Figure 14 (i). There exist $a^{\prime} \in \lambda([a]), b^{\prime} \in \lambda([b]), c^{\prime} \in \lambda([c]), d^{\prime} \in \lambda([d]), K^{\prime} \subseteq N$ and a homeomorphism $\chi:\left(K^{\prime}, a^{\prime}, b^{\prime}, c^{\prime}, d^{\prime}\right) \rightarrow\left(K_{0}, a_{0}, b_{0}, c_{0}, d_{0}\right)$.

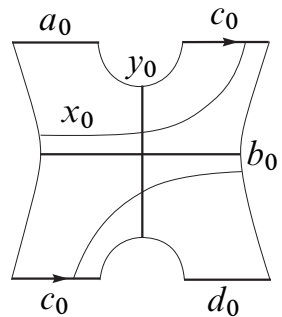

(i)

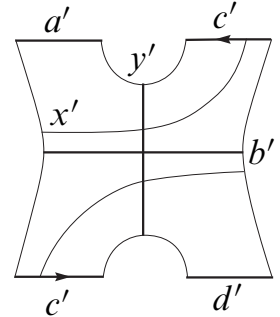

(ii)

Figure 14: Arc configurations V

Proof Let $x_{0}, y_{0}$ be as shown in Figure 14 (i). Let $x=\phi^{-1}\left(x_{0}\right), y=\phi^{-1}\left(y_{0}\right)$. Since $a, b, c, d$ are pairwise disjoint, $a, b, c$ form an embedded triangle, and $b, c, d$ form an embedded triangle, by using that $\lambda$ is injective and the results of Lemma 3.3 we can choose $a^{\prime} \in \lambda([a]), b^{\prime} \in \lambda([b]), c^{\prime} \in \lambda([c]), d^{\prime} \in \lambda([d])$ such that $a^{\prime}, b^{\prime}, c^{\prime}, d^{\prime}$ are pairwise disjoint, $a^{\prime}, b^{\prime}, c^{\prime}$ form an embedded triangle and $b^{\prime}, c^{\prime}, d^{\prime}$ form an embedded triangle. Since $y$ intersects $b$ once and $y$ is disjoint from $a, c, d$, we can choose $y^{\prime} \in \lambda([y])$ such that $y^{\prime}$ intersects $b^{\prime}$ once and $y^{\prime}$ is disjoint from $a^{\prime}, c^{\prime}, d^{\prime}$. Since $a, y, c$ form an embedded triangle, $a^{\prime}, y^{\prime}, c^{\prime}$ form an embedded triangle. Since $x$ intersects $c$ once and $x$ is disjoint from $a, b, d$, we can choose $x^{\prime} \in \lambda([x])$ such that $x^{\prime}$ intersects $c^{\prime}$ once and $x^{\prime}$ is disjoint from $a^{\prime}, b^{\prime}, d^{\prime}$. If the identification of the sides of $c^{\prime}$ is as shown in Figure 14 (ii), then we would get a contradiction by Lemma 3.3 as $a^{\prime}, x^{\prime}, b^{\prime}$ do not form an embedded triangle while $a, x, b$ form an embedded triangle. Hence, the result of the lemma follows.

Lemma 3.10 Let $a, b, c, d$ be essential, pairwise disjoint, nonisotopic, properly embedded $\operatorname{arcs}$ on $N$. Suppose that there exists a subsurface $K$ of $N$ and a homeomorphism $\phi:(K, a, b, c, d) \rightarrow\left(K_{0}, a_{0}, b_{0}, c_{0}, d_{0}\right)$ where $K_{0}$ and $a_{0}, b_{0}, c_{0}, d_{0}$ are as shown in Figure 15 (i). There exist $a^{\prime} \in \lambda([a]), b^{\prime} \in \lambda([b]), c^{\prime} \in \lambda([c]), d^{\prime} \in \lambda([d])$, $K^{\prime} \subseteq N$ and a homeomorphism $\chi:\left(K^{\prime}, a^{\prime}, b^{\prime}, c^{\prime}, d^{\prime}\right) \rightarrow\left(K_{0}, a_{0}, b_{0}, c_{0}, d_{0}\right)$. 


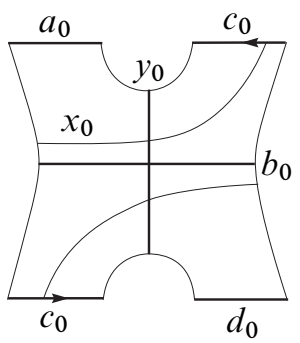

(i)

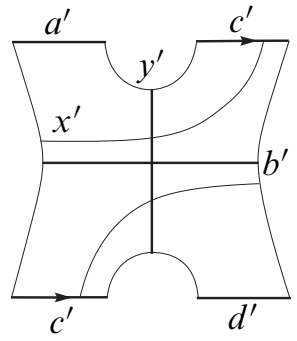

(ii)

Figure 15: Arc configurations VI

Proof Let $x_{0}, y_{0}$ be as shown in Figure 15 (i). Let $x=\phi^{-1}\left(x_{0}\right), y=\phi^{-1}\left(y_{0}\right)$. Since $a, b, c, d$ are pairwise disjoint, $a, b, c$ form an embedded triangle, and $b, c, d$ form an embedded triangle, by using that $\lambda$ is injective and the results of Lemma 3.3 we can choose $a^{\prime} \in \lambda([a]), b^{\prime} \in \lambda([b]), c^{\prime} \in \lambda([c]), d^{\prime} \in \lambda([d])$ such that $a^{\prime}, b^{\prime}, c^{\prime}, d^{\prime}$ are pairwise disjoint, $a^{\prime}, b^{\prime}, c^{\prime}$ form an embedded triangle and $b^{\prime}, c^{\prime}, d^{\prime}$ form an embedded triangle. Since $y$ intersects $b$ once and $y$ is disjoint from $a, c, d$, we can choose $y^{\prime} \in \lambda([y])$ such that $y^{\prime}$ intersects $b^{\prime}$ once and $y^{\prime}$ is disjoint from $a^{\prime}, c^{\prime}, d^{\prime}$. Since $a, y, c$ form an embedded triangle, $a^{\prime}, y^{\prime}, c^{\prime}$ form an embedded triangle. Since $x$ intersects $c$ once and $x$ is disjoint from $a, b, d$, we can choose $x^{\prime} \in \lambda([x])$ such that $x^{\prime}$ intersects $c^{\prime}$ once and $x^{\prime}$ is disjoint from $a^{\prime}, b^{\prime}, d^{\prime}$. If the identification of the sides of $c^{\prime}$ is as shown in Figure 15 (ii), then we would get a contradiction by Lemma 3.3 as $a^{\prime}, x^{\prime}, d^{\prime}$ do not form an embedded triangle while $a, x, d$ form an embedded triangle. Hence, the result of the lemma follows.

Lemma 3.11 Suppose that $(g, r) \neq(1,1),(g, r) \neq(1,2)$ and $(g, r) \neq(2,1)$. Let $\lambda: \mathcal{A}(N) \rightarrow \mathcal{A}(N)$ be an injective simplicial map of $\mathcal{A}(N)$. $\lambda$ preserves the topological equivalence of ordered triangulations on $N$ (ie for a given ordered triangulation $T=\left(a_{1}, a_{2}, \ldots, a_{3 g+3 r-6}\right)$ on $N$, and a corresponding ordered triangulation $T^{\prime}=$ $\left(a_{1}^{\prime}, a_{2}^{\prime}, \ldots, a_{3 g+3 r-6}^{\prime}\right)$ where $\left[a_{i}^{\prime}\right]=\lambda\left(\left[a_{i}\right]\right)$ for all $i=1,2, \ldots, 3 g+3 r-6$, there exists a homeomorphism $h: N \rightarrow N$ such that $h\left(a_{i}\right)=a_{i}^{\prime}$ for all $\left.i=1,2, \ldots, 3 g+3 r-6\right)$.

Proof $N_{T}$ and $N_{T^{\prime}}$ have both $2 g+2 r-4$ components. Let $\left\{\Delta_{i}: 1 \leq i \leq 2 g+2 b-4\right\}$ be the distinct components of $N_{T}$. By Lemmas 3.3, 3.4, 3.5 we know that "images" of triangles of $T$ under $\lambda$ are triangles of $T^{\prime}$, and the types of these triangles are preserved. Let $\Delta_{i}^{\prime}$ be the component of $N_{T^{\prime}}$ which corresponds to $\Delta_{i}$ under this correspondence. 
Suppose that $\Delta_{i}$ is embedded. By Lemma 3.3, $\Delta_{i}^{\prime}$ is embedded. Let $j_{i}, k_{i}$, and $l_{i}$ be the sides of $\Delta_{i}$ and $j_{i}^{\prime}, k_{i}^{\prime}$, and $l_{i}^{\prime}$ be the corresponding sides of $\Delta_{i}^{\prime}$. There exists a homeomorphism $g_{i}:\left(\Delta_{i}, j_{i}, k_{i}, l_{i}\right) \rightarrow\left(\Delta_{i}^{\prime}, j_{i}^{\prime}, k_{i}^{\prime}, l_{i}^{\prime}\right)$. The orientation type of $g_{i}$ (ie whether it is orientation-reversing or orientation-preserving) is fixed.

Suppose that $\Delta_{i}$ is regular nonembedded. By Lemma 3.4, $\Delta_{i}^{\prime}$ is regular nonembedded. Let $j_{i}, k_{i}$ be the sides of $\Delta_{i}$ such that $j_{i}$ joins two different boundary components of $\partial N$, and $k_{i}$ joins a component of $\partial N$ to itself. Let $j_{i}^{\prime}, k_{i}^{\prime}$ be the corresponding sides of $\Delta_{i}^{\prime}$. By Lemma 3.4, $j_{i}^{\prime}$ joins two different boundary components of $\partial N$, and $k_{i}^{\prime}$ joins a component of $\partial N$ to itself. There exist homeomorphisms $g_{i}, g_{i}^{*}:\left(\Delta_{i}, j_{i}, k_{i}\right) \rightarrow$ $\left(\Delta_{i}^{\prime}, j_{i}^{\prime}, k_{i}^{\prime}\right)$ such that $g_{i}$ is orientation preserving on $k_{i}$ and $g_{i}^{*}$ is orientation reversing on $k_{i}$.

Suppose that $\Delta_{i}$ is twisted nonembedded. By Lemma 3.5, $\Delta_{i}^{\prime}$ is twisted nonembedded. Let $j_{i}, k_{i}$ be the sides of $\Delta_{i}$ such that $j_{i}$ joins two different boundary components of $\partial N$, and $k_{i}$ joins a component of $\partial N$ to itself. Let $j_{i}^{\prime}, k_{i}^{\prime}$ be the corresponding sides of $\Delta_{i}^{\prime}$. By Lemma 3.5, $j_{i}^{\prime}$ joins two different boundary components of $\partial N$, and $k_{i}^{\prime}$ joins a component of $\partial N$ to itself. There exist homeomorphisms $g_{i}, g_{i}^{*}:\left(\Delta_{i}, j_{i}, k_{i}\right) \rightarrow$ $\left(\Delta_{i}^{\prime}, j_{i}^{\prime}, k_{i}^{\prime}\right)$ such that $g_{i}$ is orientation preserving on $k_{i}$ and $g_{i}^{*}$ is orientation reversing on $k_{i}$.

Lemmas 3.6, 3.7, 3.8, 3.9, 3.10 ensure that we can choose homeomorphisms $h_{i}: \Delta_{i} \rightarrow$ $\Delta_{i}^{\prime}, 1 \leq i \leq 2 g+2 r-4$, where $h_{i}$ is isotopic to $g_{i}$, if $\Delta_{i}$ is embedded, and $h_{i}$ is isotopic to either $g_{i}$ or $g_{i}^{*}$, if $\Delta_{i}$ is nonembedded, so that the unique homeomorphism $h: N_{T} \rightarrow N_{T^{\prime}}$ whose restriction to $\Delta_{i}$ is equal to $h_{i}, 1 \leq i \leq 2 g+2 r-4$, covers a homeomorphism $h: N \rightarrow N$. By Lemmas 3.6, 3.7, 3.8, 3.9, 3.10, the homeomorphisms $h_{i}: \Delta_{i} \rightarrow \Delta_{i}^{\prime}$ and $h_{j}: \Delta_{j} \rightarrow \Delta_{j}^{\prime}$ associated to embedded triangles $\Delta_{i}$ and $\Delta_{j}$ which have sides corresponding to the same element of $T$, can be isotoped by a relative isotopy to agree on that side. In other words, the restrictions of $h_{i}$ and $h_{j}$ to pairs of sides which correspond to the same element of $T$ have the same orientation type as such homeomorphisms between fixed elements of $T$ and $T^{\prime}$.

When $\Delta_{i}$ is nonembedded, this condition on compatibility of orientation types of restrictions on pairs of sides which correspond to the same element of $T$ can be realized on all such pairs by making the appropriate choice of either $g_{i}$ or $g_{i}^{*}$.

Once the correct choices are made so that this compatibility of orientations is realized, we may isotope the chosen homeomorphisms, $h_{i}$, to homeomorphisms which agree, as homeomorphisms between fixed elements of $T$ and $T^{\prime}$, on all pairs of sides which correspond to the same element of $T$. By gluing these homeomorphisms, we get a homeomorphism $h: N \rightarrow N$ which maps each element $j$ of $T$ to the corresponding element $j^{\prime}$ of $T^{\prime}$. 
Theorem 3.12 Suppose that $(g, r) \neq(1,1),(g, r) \neq(1,2)$ and $(g, r) \neq(2,1)$. If $\lambda: \mathcal{A}(N) \rightarrow \mathcal{A}(N)$ is an injective simplicial map, then it is induced by a homeomorphism $h: N \rightarrow N$ (ie $\lambda([a])=[h(a)]$ for every essential properly embedded arc $a$ on $N$ ).

Proof Let $\Delta$ be a top dimensional simplex in $\mathcal{A}(N)$. By Lemma 3.11 there exists a homeomorphism $h: N \rightarrow N$ such that $\lambda$ agrees with the map induced by $h$ on every vertex in $\Delta$. We can see that $\lambda$ agrees with this map on every vertex of $\mathcal{A}(N)$ by following the proof of Lemma 4.13 in [8], and using that (i) every vertex is contained in a top dimensional simplex, (ii) every codimension one face of a top dimensional simplex is contained in one or two top dimensional simplices, (iii) between any two top dimensional simplices $\Delta$ and $\Delta^{\prime}$ of $\mathcal{A}(N)$ there exists a chain $\Delta=\Delta_{0}, \Delta_{1}, \ldots, \Delta_{m}=\Delta^{\prime}$ of top dimensional simplices in $\mathcal{A}(N)$ connecting $\Delta$ to $\Delta^{\prime}$ such that any two consecutive simplices $\Delta_{i}, \Delta_{i+1}$ have exactly one common face of codimension 1 (by Hatcher [7]; see also Mosher's proof for the orientable case in [19]).

Theorem 3.13 Suppose that $(g, r) \neq(1,1),(g, r) \neq(1,2)$ and $(g, r) \neq(2,1)$. Then $\operatorname{Mod}_{N} \cong \operatorname{Aut}(\mathcal{A}(N))$.

Proof $\operatorname{Mod}_{N}$ acts naturally on $\mathcal{A}(N)$ by simplicial automorphisms. Let $\rho: \operatorname{Mod}_{N} \rightarrow$ $\operatorname{Aut}(\mathcal{A}(N))$ be the natural representation corresponding to this action. Every automorphism of $\mathcal{A}(N)$ is induced by a homeomorphism of $N$ by Theorem 3.12, hence $\rho$ is onto. Let $h$ be an element of $\operatorname{ker}(\rho)$ and $H: N \rightarrow N$ be a homeomorphism of $N$ representing $h$. The homeomorphism $H: N \rightarrow N$ preserves the isotopy class of every essential arc on $N$.

Since $(g, r) \neq(1,1),(g, r) \neq(1,2)$ and $(g, r) \neq(2,1)$ there exists a triangulation $T$ on $N$ which has an embedded triangle $\Delta$. Let $a, b$ and $c$ be the sides of $\Delta$. Since $(g, r) \neq(1,1),(g, r) \neq(1,2)$ and $(g, r) \neq(2,1), \Delta$ is the unique triangle of $T$ which has these sides. Since $H: N \rightarrow N$ preserves the isotopy class of every essential arc on $N$, we may isotope $H: N \rightarrow N$ to a homeomorphism $H_{0}: N \rightarrow N$ such that for each element $j$ of $T, H_{0}(j)=j$. Since $a, b$ and $c$ are elements of $T$ there exists an embedded triangle $\Delta^{\prime}$ of $T$ on $N$ with sides $H_{0}(a), H_{0}(b)$ and $H_{0}(c)$ such that $\Delta^{\prime}=H_{0}(\Delta)$. Since $a, b$ and $c$ are elements of $T$, we have $H_{0}(a)=a$, $H_{0}(b)=b$ and $H_{0}(c)=c$. Now we see that $\Delta^{\prime}$ and $\Delta$ are triangles on $N$ with sides $a, b$ and $c$, and so $\Delta^{\prime}=\Delta$. Hence, the homeomorphism $H_{0}: N \rightarrow N$ restricts to a homeomorphism $H_{0} \mid:(\Delta, a, b, c) \rightarrow(\Delta, a, b, c)$. We may isotope $H_{0}: N \rightarrow N$ relative to the union $|T|$ of the elements of $T$, to a homeomorphism $H_{1}: N \rightarrow N$ which restricts to the identity map $H_{1} \mid=\mathrm{id}_{\Delta}: \Delta \rightarrow \Delta$ of $\Delta$. 
Any other triangle $\Delta^{\prime \prime}$ of the triangulation $T$ is connected to the triangle $\Delta$ by a sequence of triangles which have sides corresponding to the same element of $T$. Since $(g, r) \neq(1,1),(g, r) \neq(1,2)$ and $(g, r) \neq(2,1)$, there are $k=2 g+2 r-4$ triangles in $T$. By a finite induction argument we can construct a sequence of homeomorphisms, $H_{i}: N \rightarrow N, 0 \leq i \leq k$, such that $H_{0}$ preserves each element of $T$ and is isotopic to $H$ on $N ; H_{1}$ preserves each element of $T$, fixes each point of at least one triangle of $T$ on $N$, and is isotopic to $H_{0}$ on $N$ relative to $|T|$; and for each integer $i$ with $2 \leq i \leq k, H_{i}$ preserves each element of $T$, fixes each point of at least $i$ triangles of $T$, and is isotopic to $H_{i-1}$ on $N$ relative to the union of $|T|$ with $i-1$ triangles of $T$ fixed pointwise by $H_{i-1}$. We have $H_{k}=\mathrm{id}_{N}: N \rightarrow N$ and $H: N \rightarrow N$ is isotopic to $H_{k}: N \rightarrow N$. So, $H: N \rightarrow N$ is isotopic to $\operatorname{id}_{N}: N \rightarrow N$. Hence, the natural representation $\rho: \operatorname{Mod}_{N} \rightarrow \operatorname{Aut}(\mathcal{A}(N))$ is an isomorphism.

Acknowledgments We thank Ursula Hamenstadt for her comments about the paper. We thank Peter Scott for some discussions about this work and Lee Mosher for his interest in the paper.

This paper was written during the author's stay at Mathematical Sciences Research Institute (MSRI) in Berkeley in Fall 2007. The author thanks MSRI for the excellent conditions provided during this stay.

\section{References}

[1] F Atalan-Ozan, Automorphisms of complexes of curves on odd genus nonorientable surfaces arXiv:math.GT/0512368

[2] J Behrstock, D Margalit, Curve complexes and finite index subgroups of mapping class groups, Geom. Dedicata 118 (2006) 71-85 MR2239449

[3] R W Bell, D Margalit, Injections of Artin groups, Comment. Math. Helv. 82 (2007) 725-751 MR2341838

[4] T E Brendle, D Margalit, Commensurations of the Johnson kernel, Geom. Topol. 8 (2004) 1361-1384 MR2119299

[5] T E Brendle, D Margalit, Addendum to: "Commensurations of the Johnson kernel" [Geom. Topol. 8 (2004), 1361-1384; MR2119299], Geom. Topol. 12 (2008) 97-101 MR2377246

[6] B Farb, N V Ivanov, The Torelli geometry and its applications: research announcement, Math. Res. Lett. 12 (2005) 293-301 MR2150885

[7] A Hatcher, On triangulations of surfaces, Topology Appl. 40 (1991) 189-194 MR1123262 
[8] E Irmak, Superinjective simplicial maps of complexes of curves and injective homomorphisms of subgroups of mapping class groups, Topology 43 (2004) 513-541 MR2041629

[9] E Irmak, Complexes of nonseparating curves and mapping class groups, Michigan Math. J. 54 (2006) 81-110 MR2214789

[10] E Irmak, Superinjective simplicial maps of complexes of curves and injective homomorphisms of subgroups of mapping class groups. II, Topology Appl. 153 (2006) 1309-1340 MR2202856

[11] E Irmak, M Korkmaz, Automorphisms of the Hatcher-Thurston complex, Israel J. Math. 162 (2007) 183-196 MR2365859

[12] E Irmak, J D McCarthy, Injective simplicial maps of the arc complex, to appear in Turkish J. Math. arXiv:math.GT/0606612

[13] N V Ivanov, Automorphisms of complexes of curves and of Teichmüller spaces, Internat. Math. Res. Notices (1997) 651-666 MR1460387

[14] M Korkmaz, Automorphisms of complexes of curves on punctured spheres and on punctured tori, Topology Appl. 95 (1999) 85-111 MR1696431

[15] M Korkmaz, Mapping class groups of nonorientable surfaces, Geom. Dedicata 89 (2002) 109-133 MR1890954

[16] F Luo, Automorphisms of the complex of curves, Topology 39 (2000) 283-298 MR1722024

[17] D Margalit, Automorphisms of the pants complex, Duke Math. J. 121 (2004) 457-479 MR2040283

[18] JD McCarthy, WR Vautaw, Automorphisms of Torelli groups arXiv: math.GT/0311250

[19] L Mosher, Tiling the projective foliation space of a punctured surface, Trans. Amer. Math. Soc. 306 (1988) 1-70 MR927683

[20] M Scharlemann, The complex of curves on nonorientable surfaces, J. London Math. Soc. (2) 25 (1982) 171-184 MR645874

[21] M Stukow, Dehn twists on nonorientable surfaces arXiv:math.GT/0601137

Department of Mathematics \& Statistics, Bowling Green State University Bowling Green, $\mathrm{OH} 43403$, USA

eirmak@bgsu . edu

http://www-math. bgsu.edu/ eirmak

Received: 13 August 2008 